Revue d'histoire de l'Amérique française

REVUE D.HISTOIRE DE L'AMÉRIQUE FRANÇAISE

\title{
L'invention du pèlerinage de la Tour des Martyrs de Saint-Célestin (1898-1930)
}

\section{Jean Roy}

Volume 43, numéro 4, printemps 1990

URI : https://id.erudit.org/iderudit/304837ar

DOI : https://doi.org/10.7202/304837ar

Aller au sommaire du numéro

Éditeur(s)

Institut d'histoire de l'Amérique française

ISSN

0035-2357 (imprimé)

1492-1383 (numérique)

Découvrir la revue

Citer cet article

Roy, J. (1990). L’invention du pèlerinage de la Tour des Martyrs de Saint-Célestin (1898-1930). Revue d'histoire de l'Amérique française, 43(4), 487-507. https://doi.org/10.7202/304837ar
Résumé de l'article

Vers 1930, les élites religieuses du diocèse de Nicolet ont réussi l'invention du pèlerinage de la Tour des Martyrs de Saint-Célestin, au Québec. Afin d'expliquer le mythe fondateur, les promoteurs s'appliquèrent à répondre aux questions sur l'origine du sanctuaire : le fondateur, Calixte Marquis, les reliques et le merveilleux qui leur est rattaché, ainsi que l'eau miraculeuse. L'objectif de cet article est d'analyser cette création, en tâchant de la situer dans son contexte. 


\title{
L'INVENTION DU PÈLERINAGE DE LA TOUR DES MARTYRS DE SAINT-CÉLESTIN (1898-1930) ${ }^{1}$
}

\author{
JEAN ROY \\ Centre d'études québécoises \\ Université du Québec à Trois-Rivières
}

\section{RÉSUMÉ}

Vers 1930 , les élites religieuses du diocèse de Nicolet ont réussi l'invention du pèlerinage de la Tour des Martyrs de Saint-Célestin, au Québec. Afin d'expliquer le mythe fondateur, les promoteurs s'appliquèrent à répondre aux questions sur l'origine du sanctuaire: le fondateur, Calixte Marquis, les reliques et le merveilleux qui leur est rattaché, ainsi que l'eau miraculeuse. L'objectif de cet article est d'analyser cette création, en tâchant de la situer dans son contexte.

\section{ABSTRACT}

In the early thirties, the religious leaders of the diocese of Nicolet invented a place of pilgrimage called La Tour des Martyrs in the parish of Saint-Célestin. In order to explain the myth behind this shrine, the promoters devoted considerable effort to answering questions regarding its founder, Calixte Marquis, the relics and their aura of holy mystery, the miraculous springwater. In the present article, the author attempts to analyse this work of edification within its social context.

La piété ultramontaine, appelée aussi piété romaine, se caractérise par la place exceptionnelle accordée au culte marial, à l'Enfant-Jésus, à saint Joseph et à sainte Anne, aux dévotions christologiques: chemins de croix et culte du Sacré-Coeur ainsi qu'au culte des saints ultramontains comme sainte Philomène ${ }^{2}$. Dans cette religion iconique, le corps, la relique, l'image sont autant de signes dont on cherche la présence

1 Les travaux de recherche que je mène sur le changement religieux au XXe siècle sont subventionnés par le Conseil de recherches en sciences humaines du Canada. Je remercie mes collègues René Hardy et Serge Gagnon pour leurs conseils.

2 Sur la piété ultramontaine, voir Guy Laperrière, «Religion populaire, religion de clercs? Du Québec à la France, 1972-1982», B. Lacroix et J. Simard, dir., Religion populaire, religion de clercs? (Québec, Institut québécois de recherche sur la culture, 1984), 21-51. Parmi les travaux cités dans la bibliographie de cet ouvrage, j'insiste sur ceux de Y.-M. Hilaire, Une crirétienté au XIXe siècle? la vie religieuse des populations du diocèse d'Arras (1840-1914) (Villeneuve-d'Ascq, Publications de l'Université de Lille III, 1977), 492 p., et de Michel Lagrée, «Religion populaire et populisme religieux au XIXe siècle», J. Delumeau, dir., Histoire vécue du peuple chrétien (Toulouse, Privat, 1979), 2: 157-178. Sur le culte rendu à sainte Philomène, voir Pierre Savard, "La dévotion à sainte Philomène», Aspects du catholicisme canadien-français au XIXe siècle (Montréal, Fides, 1986), 173-191. 
tangible. Aucun endroit ne les offre mieux à la vénération que le lieu hautement symbolique qu'est le pèlerinage ${ }^{3}$.

Le mouvement d'implantation des pèlerinages au Québec est à mettre en relation avec la poussée de la piété ultramontaine. Avec douze des vingt fondations de pèlerinages au XIXe siècle, les années 18731883 composent un des deux temps forts de ce mouvement, l'autre se situant entre 1947 et 1955 . De ces douze nouveaux lieux sacrés, six sont dédiés à la Vierge (Notre-Dame), trois à sainte Anne, deux à saint Joseph, un au Sacré-Coeur. Ces dédicaces traduisent admirablement la diffusion des cultes romains. La faible représentation du «saint ordinaire» a déjà été notée $e^{4}$. Il n'y a pour les 85 sanctuaires que neuf consécrations à leur dévotion. Le sanctuaire de la Tour des Martyrs de SaintCélestin (1898) est de ce nombre, le seul, d'ailleurs, avant le XXe siècle.

Pendant plus de vingt-cinq ans, il s'agit tout au plus d'un modeste lieu de prière fréquenté principalement, mais non exclusivement, par les paroissiens de Saint-Célestin. Or, en 1930, le sanctuaire de la Tour des Martyrs a acquis une grande crédibilité auprès des fidèles qui y viennent en grand nombre pour vénérer les reliques des saints et les intercesseurs qu'on leur propose comme modèles ${ }^{5}$. Pour mousser la conviction, les promoteurs du sanctuaire ont dû fournir des réponses à un certain nombre de questions et, de la sorte, imposer ce pèlerinage inventé de toutes pièces par les élites religieuses. Le clergé s'est donné pour tâche de créer le mythe fondateur, de le faire connaître et de susciter des adhésions. La question des origines prend dès lors une place considérable et les promoteurs s'appliquent à son explication dans $L e$ Guide du pèlerin à la Tour des Martyrs de St-Célestin ${ }^{6}$. Qui est le fondateur du sanctuaire? Où a-t-il amassé les reliques? Sont-elles authentiques? Ont-elles donné des signes manifestes d'intervention divine? Leur vénération a-t-elle donné lieu à des miracles? Le sanctuaire de la Tour des Martyrs fut-il le théâtre de prodiges? Le Guide cherche à satisfaire ces curiosités et «à justifier la dévotion par l'histoire» ${ }^{7}$.

3 Sur le pèlerinage, voir Alphonse Dupront, Du sacré. Croisades et pèlerinages. Images et langages (Paris, Gallimard, 1987), 315-415, et Pierre Boglioni et Benoît Lacroix, dir., Les pèlerinages au Québec (Québec, Presses de l'Université Laval, 1981), 160 p.

4 Voir Guy Laperrière, «Les lieux de pèlerinage au Québec: une vue d'ensemble», P. Boglioni et B. Lacroix, op. cit., 29-64.

5 Jean Roy, «Mutation et déclin de la solidarité religieuse: la fréquentation de la Tour des Martyrs de Saint-Célestin», Roger Levasseur, dir., De la sociabilité, spécificité et mutations (Montréal, Boréal, 1990), 121-135.

6 Abbé Georges Désilets, Le guide du pèlerin à la Tour des Martyrs de Saint-Célestin (Québec, Le Soleil, 1932), 87 p. (désormais, Le Guide). Le nom de La Tour des Martyrs est emprunté à un édifice situé dans la ville de Ramley, sur la route de Jaffa à Jérusalem: «La Tour des Quarante Martyrs», cité dans Le Guide, 24.

7 Claire Dolan, «Jalons pour une historiographie des pèlerinages au Québec», P. Boglioni et B. Lacroix, op. cit., 65-95. 
Pour l'étude de la culture religieuse au XXe siècle, Le Guide est, à coup sûr, un document d'une grande richesse qui, notons-le, connaît une bonne diffusion. Outre qu'il est entre les mains des pèlerins, il sert de source d'information aux Soeurs Grises qui accueillent et guident les visiteurs; par ailleurs, la presse en reproduit des extraits. Dans le présent article, nous prêterons une grande attention à l'outil de propagande qu'est Le Guide, car il se présente comme le résultat d'un travail susceptible d'emporter la conviction du lecteur et de stimuler sa ferveur. Il offre à la réflexion du pèlerin, tout comme le sanctuaire, des dévotions qui s'insèrent dans une culture religieuse bien intégrée à la culture générale, du moins se présente-t-il ainsi.

En 1930, dans le diocèse de Nicolet, on a réussi l'invention d'un pèlerinage. L'objectif de cette étude est d'analyser cette création, la situant dans son contexte et, par là, chercher à le comprendre ${ }^{8}$.

\section{I - À L'ORIGINE DU PÈLERINAGE: UN PROMOTEUR CONTROVERSÉ}

Deux auteurs ont fait principalement la promotion du pèlerinage, l'abbé Georges Désilets, directeur du sanctuaire, lorsqu'il rédige $L e$ Guide et l'abbé Arthur Girard, professeur de philosophie au Séminaire de Nicolet. Le 24 mai 1924, Girard signe un article substantiel dans L'Action catholique. Ce texte, abondamment illustré, intitulé «La Tour des Martyrs de Saint-Célestin», subit de légères retouches, pour prendre enfin la forme d'un petit livre édité à trois reprises ${ }^{9}$. Originaire de la paroisse de Saint-Célestin, Girard connaît l'oeuvre de Calixte Marquis, fondateur du lieu de prière, décédé en 1904. Pour se renseigner davantage, il a la possibilité de consulter les papiers laissés par Marquis et d'interroger les nombreuses personnes qui l'ont côtoyé. Dans ce but, il s'adresse à soeur Saint-Antoine (1874-1933), une des trois Soeurs Grises qui, en mission à Saint-Célestin depuis 1898, résident dans la maison de Marquis.

Aux yeux d'Arthur Girard, la soeur Saint-Antoine est un témoin exceptionnel, sinon unique, car il peut présumer qu'elle a entendu Marquis raconter des faits jusque-là inédits concernant la réelle identité du fondateur de la Congrégation des Soeurs de l'Assomption et l'histoire des reliques. C'est ainsi que la religieuse rédige, à la demande de Girard, ses mémoires sur la fondation des Soeurs de l'Assomption et sur la Tour des Martyrs ${ }^{10}$. Le texte est remis en deux parties, les 12 février et 12 avril 1928. Si Girard ne puise pas beaucoup dans ce mémoire, bien

$8 \quad$ Pour les fins de l'étude, j'ai consulté les archives des Soeurs Grises de Montréal, à Nicolet (ASGN), celles du Séminaire de Nicolet (ASN), de l'évêché de Nicolet (AEN), de la paroisse de Saint-Célestin, des Soeurs de l'Assomption de Nicolet (ASA) et celles du Séminaire de TroisRivières (ASTR).

9 Arthur Girard, La Tour des Martyrs de Saint-Célestin (Québec, Imprimerie Ernest Tremblay, 1931), 96 p., 3e édition. Saint-Célestin, comté de Nicolet.

${ }_{10}$ ASGN. Manuscrit à monsieur l'abbé Arthur Girard, prêtre, 39 p. 
qu'il soit amené à apporter une correction au sujet des reliques de sainte Anne, il n'en va pas de même de Georges Désilets, l'auteur du Guide du pèlerin à la Tour des Martyrs de St-Célestin. Grâce au témoignage de la religieuse, Désilets peut donner la parole à Marquis. Le récit de soeur Saint-Antoine et les archives laissées par Marquis, peu utilisées par Girard, lui permettent de présenter le fondateur du pèlerinage.

Ni Girard ni Désilets ne désirent écrire une biographie, même très courte, de Calixte Marquis ${ }^{11}$. L'un et l'autre admettent que «le mouvement de sa vie n'a pas toujours été agréable à tout le monde» et que, en retracer le cours est «une tâche délicate». Ils sont ainsi d'accord avec Herman Brunault, coadjuteur de l'évêque de Nicolet, qui, à l'occasion de la nouvelle année 1900, souhaite à Marquis «une vieillesse heureuse, sans infirmité» et ajoute: "vous avez fait le beau temps et la pluie, un peu à votre guise dans votre vie, il faut espérer que vous saurez maintenant dissiper tous les nuages qui pourraient assombrir votre existence. ${ }^{12}$ La chronologie de la vie de Marquis (1821-1904) que l'on peut lire dans Le Guide donne les dates du «beau temps»: celles de la colonisation des paroisses du sud du comté de Nicolet et celles du comté d'Arthabaska. Marquis dessert les missions à partir du mois de juillet 1845; en octobre de la même année, il est nommé vicaire de Charles Harper dans la paroisse de Saint-Grégoire; il y réside jusqu'en 1854, puis il va s'installer dans la paroisse de Saint-Célestin dont il est le curé à partir de 1852. De là, il part pour les missions, dont certaines sont situées près du lac Aylmer, à plus de 100 kilomètres de sa paroisse.

Le nom de Marquis rappelle à tous cette période pas très éloignée de la colonisation des Bois-Francs dont la mémoire collective conserve le souvenir ${ }^{13}$. La chronologie l'assimile à «un autre héroïque pionnier», l'abbé C.-E. Bélanger, son confrère de mission dans le canton de Somerset qui meurt noyé dans la redoutable savane de Stanfold, en 1845. En fait, peu de gens connaissent autant cette région qu'il arpente dans tous les sens, délimitant le territoire des nouvelles paroisses. Cette expérience l'autorise à écrire un Mémoire sur la colonisation des terres incultes du Bas-Canada ${ }^{14}$. Marquis croit que l'institution paroissiale produit un effet dynamique sur la colonisation et il en est de même du diocèse en qui il reconnaît un instrument de la colonisation. En consé-

11 Ce n'est pas non plus le but ici. On trouvera une bibliographie ainsi que le repérage des sources utiles dans un article en trois parties rédigé par Michel Morin, «Calixte Marquis», «La pensée colonisatrice de Calixte marquis» et «Calixte Marquis, missionnaire-colonisateur du canton d'Aston (1850-1867)», Les cahiers nicolétains, 3,1-2-3 (1981).

12 ASN. Succession Marquis, Brunault à Marquis, 7 janvier 1900.

13 Souvenir soigneusement entretenu, faut-il le dire, par des publications. Ainsi, C.-E. Mailhot, Les Bois-Francs (Arthabaska, Imprimerie d'Arthabaska, 1914), 4 vol. Nouvelle édition A. Fleury, 1968.

${ }_{14}$ Mémoire sur la colonisation des terres incultes du Bas-Canada pour être présenté à nos seigneurs les évêques de la province ecclésiastique du Canada réunis à Québec à l'occasion de la consécration de sa grandeur Mgr J.-L. Langevin, évêque de Rimouski, Québec, Typographie A. Côté, 1867, 18 p. 
quence, Marquis souhaite la création d'un évêché plus près de la région en voie de colonisation. Affirmant que le développement de l'agriculture a besoin de fermes modèles, Marquis désire que le Séminaire de Québec en exploite une à Saint-Wenceslas. Notons que ces idées sont aussi celles d'Antoine Labelle ${ }^{15}$. Quand celui-ci, alors sous-ministre de l'agriculture dans le gouvernement Mercier, songe, en 1889, à un moyen de promouvoir la colonisation dans la partie située au nord du lac SaintJean, il fait appel à Marquis. Tous deux travaillent à l'établissement à Mistassini des trappistes qui doivent jouer le rôle d'enseignants agricoles.

Les projets de Labelle et de Marquis d'établir un évêché connaissent toutefois des sorts différents. Le «roi du Nord» ne fait pas le poids devant l'évêque de Montréal, Mgr Fabre, et ne parvient pas à faire diviser son diocèse. Quant à Marquis, en 1882, il est engagé à fond dans la création du diocèse de Nicolet depuis que l'évêque Laflèche l'a forcé à démissionner de sa cure en 1878. Il s'y livre sans retenue, furieusement, utilisant à l'occasion un vocabulaire plus ou moins diffamatoire. Une partie de l'épiscopat québécois l'encourage et alimente sa plume aussi habile qu'acerbe. Dans ses ripostes, Laflèche ne manque pas de mots cruels à l'endroit de Marquis qu'il traite de faussaire ${ }^{16}$ et de spéculateur foncier ${ }^{17}$. Au-dessous de ces têtes d'affiche, les prêtres sollicités se divisent. Au-dessous encore, le troupeau des fidèles a peu à dire à propos de ce qu'il entend parfois au hasard d'une visite épiscopale. On suppose que cette querelle de clercs le touche faiblement. Toutefois, ces fidèles ressentiront bientôt les effets bénéfiques de la proximité d'un évêque qui intervient auprès des pouvoirs politiques dans le but de favoriser, par exemple, la construction d'un chemin de fer vers Drummondville. En effet, Marquis, le contestataire du pouvoir situé au nord du fleuve, à l'égal de Labelle devant Fabre, a réussi, lui, à démembrer le diocèse de Trois-Rivières au profit de la nouvelle circonscription de Nicolet. Cela seul mérite d'être retenu.

En 1930, le clergé, qui voit dans la colonisation le moyen d'enrayer l'exode rural, peut qualifier Marquis de héros du mouvement. De son vivant, ce dernier s'était battu contre l'émigration en NouvelleAngleterre. Des témoignages éloquents lui ont été rendus en 1882, à l'occasion de son départ pour Rome, par des hommes publics importants ainsi que par des clercs: Gédéon Ouimet, P.-J.-O. Chauveau, F. Langelier, maire de Québec, M.-E. Méthot, supérieur du Séminaire

\footnotetext{
15 Gabriel Dussault, Le curé Labelle. Messianisme, utopie et colonisation au Québec, 18501900 (Montréal, Hurtubise HMH, 1983), 392 p.

16 ASTR. Lettre de Marquis à Dominique Racine, 11 septembre 1885. De son côté, Marquis, pointant Laflèche, affirme tenir des preuves sur la réelle identité du faussaire.

Nive Voisine, Louis-François Laflèche. Deuxième évêque de Trois-Rivières (Saint-Hyacinthe, Edisem, 1980), 1: 276. Michel Morin, «La pensée colonisatrice de Calixte Marquis», loc cit., sans doute trop prudent, il n'ose confirmer l'accusation de Laflèche.
} 
de Québec, le généalogiste Cyprien Tanguay, confrère d'études. Chacun soulignait son oeuvre colonisatrice et ses travaux en faveur de l'instruction publique.

Dans ces conditions, l'absence dans Le Guide d'une mention des actions de Marquis sur ce dernier point paraît curieuse. Georges Désilets affirme qu'il a mis «la main à la cause de l'éducation de notre jeunesse», mais il ne juge pas nécessaire de rappeler autrement son action dans «la guerre des éteignoirs», ou son rôle dans la fondation des Soeurs de l'Assomption. Il est vrai que les deux sujets sont délicats. Comment, en effet, ne pas vexer une partie de la population en reconnaissant à la suite de P.-J.-O. Chauveau qu'il a «fait beaucoup pour combattre les ennemis de l'éducation de 1846 à $1851 »$ et en soulignant ses efforts pour réanimer le système scolaire, là où il était prêtre ${ }^{18}$. Prudent, Désilets n'ajoute aucune précision sur ces faits et il se garde bien d'interpréter les événements qui ont profondément divisé les fidèles. L'absence de commentaire sur la fondation de la Congrégation des Soeurs de l'Assomption n'étonne pas moins. À maintes reprises, Marquis affirme qu'il est Fundator Congregationes Sororum a Beata Virgine Assumpta. Ce titre figure d'ailleurs en bonne place dans les «authentiques» (lettres testimoniales, voir infra) qu'il délivre. En 1904, l'évêque de Trois-Rivières, François-Xavier Cloutier, en contresignant

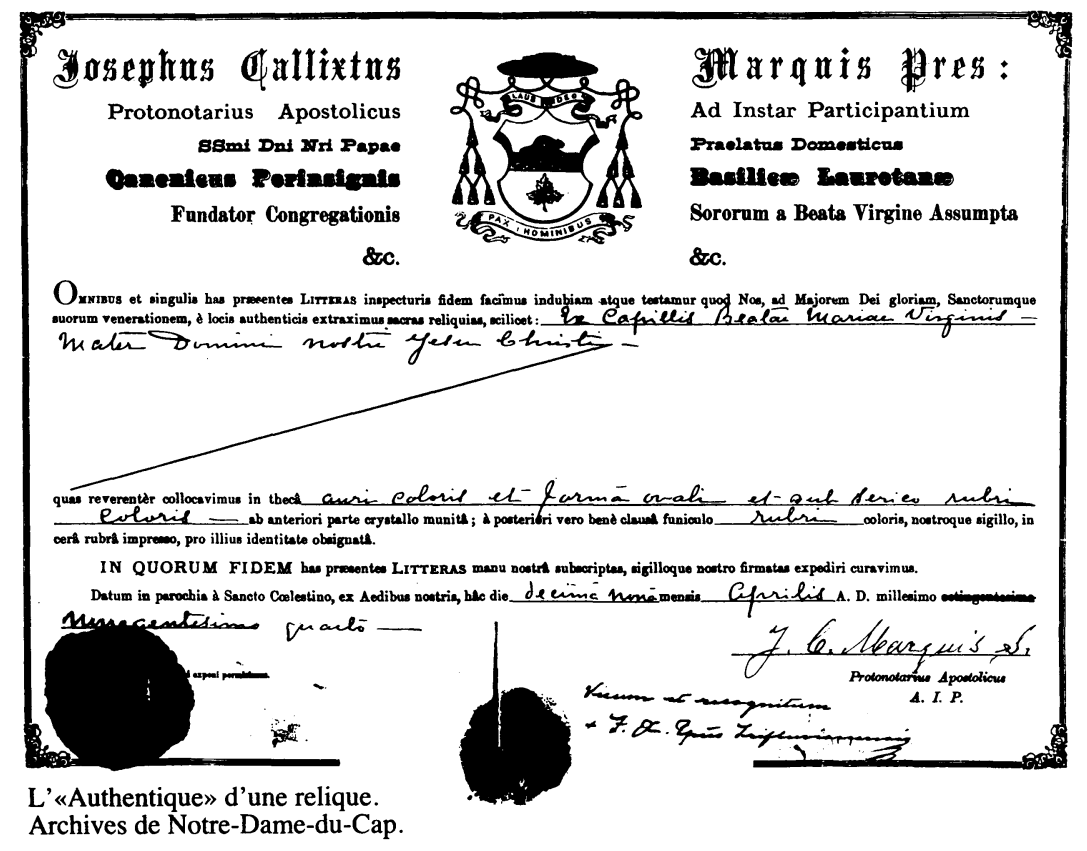

18 ASN. Succession Marquis, P.-J.-O. Chauveau à Marquis, Montréal, 7 janvier 1900. 
les «authentiques», paraît d'accord sur ce point (voir le document). Pourtant, au lendemain du décès de Marquis, on est incapable de trancher qui, de lui ou de Harper, est le réel fondateur. Interrogée, soeur Saint-Antoine appuie la thèse favorable à Marquis. Arthur Girard est si peu convaincu qu'il n'en souffle mot dans son histoire de la Tour des Martyrs. Georges Désilets garde la même discrétion sans se douter que l'image même que l'on propage du fondateur risque d'en souffrir. Quoi qu'il en soit, les fidèles n'ont que faire de ces préoccupations et restent assez peu sensibles à ces querelles de savants ${ }^{19}$.

Diversement apprécié de son vivant, Marquis ne laisse personne indifférent. Il est convaincu d'avoir fait le diocèse de Nicolet, avec Dominique Racine, l'évêque de Chicoutimi, à qui il écrit, le 11 septembre 1885: «En décembre 1882 et en janvier 1883, si nous n'eussions pas été à Rome tous deux, il n'y aurait pas, ni n'y aurait eu de diocèse de Nicolet.» ${ }^{20}$ Cet homme est-il plus craint qu'estimé ou aimé? Est-il significatif qu'aucun évêque en titre n'assiste à ses funérailles? Pas même le coadjuteur Brunault qui, pourtant, soutient l'oeuvre de Marquis. S'étonnera-t-on de savoir que son oraison funèbre marque les débuts de la querelle sur l'identité du fondateur de la Congrégation des Soeurs de l'Assomption?

Sur ces questions, l'auteur du Guide se fait plutôt silencieux. Il passe rapidement au coeur du sujet: le culte des reliques.

\section{2 - LES RELIQUES ${ }^{21}$}

\section{A - L'acquisition du trésor}

Selon son propre témoignage, Calixte Marquis voue un culte aux reliques depuis son enfance. Cette ferveur est du reste partagée par l'élite cléricale. En 1875, un professeur de la faculté de théologie de l'Université Laval, Louis-Nazaire Bégin, se fait le défenseur de ce culte

19 G. Lesage, Les origines des Soeurs de l'Assomption de la Sainte Vierge (Nicolet, Éditions A.S.V., 1957), 342 p. Lesage tire à boulets rouges sur Marquis qui, lit-on dans la correspondance d'un curé de Saint-Célestin, restait suspect. De son côté, Michel Morin, dans les articles cités, voit en Marquis un associé indispensable de Harper. L'album officiel publié à l'occasion du centenaire du diocèse, Le diocèse de Nicolet 1883-1985, 249, donne Harper comme fondateur, secondé par Marquis.

${ }_{20}$ ASN. Succession Marquis, Marquis à Dominique Racine, 11 septembre 1885.

21 Dictionnaire de droit canonique (Paris, Librairie Letouzey et Ané, 1965), article «Reliques», col. 2312-2376; et Jean-Marie Allard, Reliques et reliquaires limousins (Limoges, Bibliothèque municipale, 1988), $48 \mathrm{p}$. Le mot relique désigne les restes mortels des saints et, au sens large, les objets qui leur ont appartenu ou qui ont été en contact avec leurs corps. La distinction est faite entre les reliques insignes et les reliques simples. «On appelle reliques insignes le corps, la tête, le bras, l'avant-bras, le coeur, la main, la jambe ou la partie du corps dans laquelle le saint a souffert le martyre pourvu que cette partie soit entière et ne soit pas petite.» La relique brachiale de sainte Anne et le corps entier de saint Félix entrent dans cette catégorie. Les autres reliques sont dites simples: par exemple, un morceau de vêtement de saint Joseph, un cheveu de la Vierge. Voir aussi Nicole Hermann-Mascard, Les reliques des saints. Formation coutumière d'un droit (Paris, Klincksieck, 1975), 446 p. (chapitre 11, «L'identification des reliques»). Elle distingue entre reliques réelles et reliques représentatives. 
contre les protestants ${ }^{22}$. Vingt ans plus tard, au nom du cardinal Taschereau, dont il est le coadjuteur, il remet au supérieur du Séminaire de Québec une relique de sainte Anne acquise à Apt par Calixte Marquis. Des indices montrent que l'épiscopat québécois a suivi les décrets des pères du Quatrième Concile, sur leur vénération, en $1868^{23}$. À l'exemple de l'évêque de Saint-Hyacinthe, celui de Nicolet, dont la cathédrale voit son trésor de reliques enrichi par Calixte Marquis, veille à la diffusion de leur dévotion dans son diocèse. Il se préoccupe de leur conservation ainsi que de leur authenticité ${ }^{24}$.

Si l'élite cléricale donne le ton, de son côté le clergé paroissial cherche à se procurer des reliques afin de pourvoir les nouvelles paroisses et les lieux sacrés qu'il fonde. C'est l'interprétation qu'il faut donner à cet extrait d'une lettre que Marquis adresse, depuis Rome, à Dominique Racine, le 31 août 1883: "Si vos diocésains veulent avoir des reliques pour leurs églises paroissiales (les patrons) je pense que je puis réussir à en avoir un bon nombre. J'ai trouvé une fissure pour pénétrer dans quelques trésors». C'est en tant que pourvoyeur de reliques que l'action de Marquis est originale. Il s'emploie avec zèle à satisfaire une demande qui est grande ${ }^{25}$. La facilité avec laquelle il acquiert les reliques et l'ampleur de la réserve qu'il constitue sont étonnantes.

En effet, l'acquisition d'un nombre aussi considérable de reliques, 6200 d'après Le Guide, ne manque pas de surprendre, car, à l'époque où Marquis arrive à Rome, au mois de décembre 1882, le clergé est prévenu «contre les trafiquants de reliques tirées des catacombes ${ }^{26}$ ». Cette mise en garde du 17 janvier 1881 fait suite au décret de Léon XIII qui, le 21 décembre 1878, en condamne le commerce. Ces promulgations ont-elles pour effet de rendre plus difficile leur obtention? L'évêque de Trois-Rivières, Louis-François Laflèche, l'affirme ${ }^{27}$. Or, c'est à ce moment même que Marquis s'approprie la majeure partie du stock des reliques.

Comment le fournisseur de reliques obtient-il ses pièces? La réponse à cette question sera insatisfaisante, car l'unique source des informa-

\footnotetext{
22 L.-N. Bégin, Le culte catholique ou exposition de la foi de l'Église romaine sur le culte dû aux saints et à leurs reliques, à la bienheureuse Vierge Marie, aux images (Québec, Typographie Augustin Côté, 1875), 181 p.

${ }_{23}$ Jacques Grisé, Les conciles provinciaux de Québec et l'Église canadienne (1851-1886) (Montréal, Fides, 1979), 240.

24 AEN. Le questionnaire du rapport annuel de l'année 1896: «A-t-on les authentiques des saintes reliques?». Archives de l'évêché de Saint-Hyacinthe. Rapport-état du diocèse, 1878.

${ }_{25}$ Par analogie, la christianisation de nouvelles régions à l'époque mérovingienne va multiplier les transferts des reliques et certainement conduire à des abus. Voir Nicole HermannMascard, op. cit., 57-61.

${ }_{26}$ Dictionnaire de droit canonique (Paris, Librairie Letouzey et Ané, 1935-1965), tome 4, article «Reliques», col. 569-574; et Hippolyte Delehaye, Cinq leçons sur la méthode hagiographique (Bruxelles, Société des Bollandistes, 1934), 115 (chapitre 4, «Les reliques des saints»).

27 Archives des Ursulines de Trois-Rivières, 11, A, 2,2,84. Laflèche à Révérende Soeur Marie du Précieux-Sang, 31 mai 1884. Celle-ci lui avait demandé de lui procurer une relique.
} 
tions est Calixte Marquis lui-même. Celui-ci ouvre la voie à deux interprétations sur la provenance des reliques: la chapelle des Odeschalchi et les catacombes. D'un côté, il insiste sur l'heureuse fortune qui le fait descendre à l'hôtel tenu par un ancien chapelain de la famille Odeschalchi. Ce dernier vient d'être remercié de ses services par des maîtres passés à la franc-maçonnerie. Il raconte à Marquis qu'en guise de remerciement, ses maitres lui ont remis le trésor des reliques de la chapelle familiale, jadis amassé par un ancêtre. Cette famille, d'où proviennent le pape Innocent XI (1677-1690) et le cardinal Odeschalchi (1786-1841), s'était fort souciée d'enrichir le trésor qui finit par devenir «un ossuaire privilégié». En 1882, ce trésor fait la convoitise de Marquis. Celui-ci voit enfin sa demande satisfaite par le chapelain, fortement ému par le portrait de la chaleureuse dévotion aux reliques des saints pratiquée par les Canadiens.

Cette version est adoptée par les abbés Girard et Désilets qui ajoutent, sans plus de détail, que Marquis complète ailleurs sa collection de reliques à l'occasion de voyages, notamment en Terre-Sainte ${ }^{28}$. Ces deux auteurs ne font pas mention de reliques en provenance des catacombes, ce qui paraît curieux, car Arthur Girard, auteur de La Tour des Martyrs de Saint-Célestin, a en mains, en avril 1928, le récit de soeur Saint-Antoine qui traite de la question de l'origine des reliques.

Ce récit fonde la seconde interprétation. La religieuse ne dit rien de la chapelle des Odeschalchi. Par contre, elle accorde une grande place aux catacombes, en particulier celle de Saint-Calixte, pour laquelle Marquis aurait disposé d'une carte d'exploration. Fort du consentement des gardiens qui le laissent passer, poursuit-elle, «il a puisé en masse [...] autant qu'il a pu afin de satisfaire sa soif de posséder le plus grand nombre de reliques possible».

Cette version des faits n'est pas invraisemblable. Grâce aux fouilles archéologiques entreprises à Rome dès le début du XIXe siècle, une masse considérable de reliques sont disponibles. Entre 1800 et 1840, beaucoup d'entre elles, «douteuses», sorties des catacombes, sont répandues avec la permission plus ou moins expresse des congrégations romaines ${ }^{29}$. Par ailleurs, un grand nombre de reliques sont accumulées dans les patrimoines familiaux, comme celui des Odeschalchi. Veut-on s'en convaincre? Relisons le Martin Luther de Lucien Febvre qui donne un portrait saisissant de la chapelle de l'électeur de Saxe, le protecteur du réformateur.

\footnotetext{
28 ASN. Succession Marquis, lettres de Frédéric de Ghyvelde à Marquis, 10, 11 et 19 septembre 1896. Le Père Frédéric espérait «élever les beaux monuments de la Terre Sainte» à Saint-Célestin. Il remet à Marquis plusieurs reliques. De son côté, Marquis lui donne une relique de saint François-Xavier.

${ }^{29}$ Article «reliques» dans le Dictionnaire de théologie catholique, col. 2372.
} 
Entre ces deux interprétations, il est, à toutes fins utiles, impossible de trancher. Cependant, un peu comme Dona Rosa dans le roman de Lampedusa, il est vraisemblable que Marquis s'approvisionnait à toutes les sources qui s'offraient ${ }^{30}$.

La même imprécision subsiste au sujet de l'acquisition des reliques, fort recherchées, de sainte Anne. Marquis réussit à s'en procurer plusieurs, à Rome et à Apt, qu'il distribue ensuite avec une générosité que Désilets souligne dans Le Guide. Un chapitre intitulé "Nos Saintes Reliques à l'étranger» nomme, entre autres bénéficiaires: le cardinal Taschereau, l'église Saint-Joseph de Chicago, le curé Tétreau de l'église Saint-Jean-Baptiste de New York, l'église Saint-Jean-Baptiste de New York et le pèlerinage de Sainte-Anne-de-Beaupré. Les reliques des trois derniers donataires méritent une attention particulière, car les promoteurs furent amenés à trancher à propos de leur origine. Où Marquis les a-t-il prises? Et qu'en dit-il?

Les Annales de la Bonne Sainte Anne relatent les événements qui entourent la translation de la fameuse relique au sanctuaire ${ }^{31}$. Le texte est de Marquis qui raconte les péripéties de sa mission à Rome: son départ et l'acquisition du fragment du bras de sainte Anne, grâce à l'intervention du pape Léon XIII auprès de l'abbé de la basilique SaintPaul-hors-les-Murs. Il fait ensuite le récit de la vénération dont elle fut l'objet à New York, à l'occasion de son exposition dans l'église SaintJean-Baptiste. Enfin, il décrit le miracle qui s'y produisit, miracle dont elle tire sa grande notoriété. Remarquons que le compte rendu établit de façon indiscutable l'origine de la relique. Toutefois, il ne dit rien des deux autres.

La question fut posée à soeur Saint-Antoine. Dans son mémoire, la religieuse rappelle qu'une soeur a interrogé Marquis à ce sujet. En guise de réponse, l'octogénaire affirma plus d'une fois qu'il les avait

prises dans son tombeau même qui se trouve dans la Basilique de Sainte-Anne d'Apte [sic] en France. Au moyen d'une petite scie, j'ai scié moi-même mes trois reliques que j'ai détachées du bras de la bonne sainte Anne, non pas sans émotion. Pendant que j'en avais (embelle) [sic] j'en ai profité, et je me suis servi comme il faut. $^{32}$

À son arrivée à New York, poursuit-elle, Marquis donne l'une d'entre elles au curé Tétreau, il réserve la seconde au sanctuaire de

30 Dona Rosa connaît les paroisses en difficultés financières ou encore une noble famille en décadence. C'est là qu'elle va chercher les reliques. Pourvues des preuves d'authenticité, les reliques sont ensuite posées dans les reliquaires, puis installées dans la chapelle familiale qui en contient bien soixante-quatorze. Lampedusa, Le Guépard (Paris, Seuil, 1979), 234-235.

31 «La relique insigne envoyée par Léon XIII», Annales de la Bonne Ste-Anne-de-Beaupré, 20,6 (septembre 1892): 101-115.

32 ASGN. Manuscrit, à monsieur l'abbé Arthur Girard, prêtre, 39 p. 
Sainte-Anne-de-Beaupré et il garde la troisième qu'il apporte à SaintCélestin. Comme si elle reconnaissait l'importance de la question, l'auteure du mémoire répète ses informations.

Arthur Girard est-il convaincu par l'assurance de la religieuse? Partiellement, ainsi qu'on va le voir. Dans les deux premières éditions de l'histoire de la Tour des Martyrs, il note que Marquis «apportait avec lui, de Rome, une insigne relique de sainte Anne [...]», provenant de la basilique de Saint-Paul-hors-les-Murs. L'édition de 1931, la troisième, ajoute une note correctrice disant que Marquis a alors en sa possession trois reliques. Il remet «la plus considérable» au curé de l'église Saint-Jean-Baptiste de New York, Frédéric Tétreau; la deuxième appartient déjà aux Rédemptoristes; quant à la troisième, elle prend le chemin de Saint-Célestin. Girard fait donc sienne cette partie de la relation de la religieuse sur les trois reliques qui, en 1892, sont à New York. Par contre, il néglige totalement cette autre partie qui traite de l'origine.

Comment Désilets tranche-t-il? S'agissant de la relique donnée à Tétreau, Désilets s'appuie sur une lettre dans laquelle le curé de l'église canadienne-française de New York déclare avoir reçu de Marquis «l'authentique d'une relique de Ste-Anne (et la) délibération des Fabriciens d'Apt, à l'égard de cette relique» ${ }^{33}$. Il sait également que Tétreau la donna, en 1901, à l'évêque Gravel qui l'incorpora dans le diocèse de Nicolet. Alors que l'évêque signe, à cette occasion, une lettre circulaire pour marquer sa reconnaissance à l'endroit du généreux donateur, Marquis se montre ulcéré par le geste, car il estime que la relique doit lui revenir $^{34}$. La conclusion s'impose d'elle-même à Désilets: il écrit que Marquis l'a «obtenue à Apt».

L'origine de celle qui était destinée aux Rédemptoristes ne semble pas soulever de difficulté. Il n'accorde aucune crédibilité aux déclarations de la religieuse, leur préférant d'emblée le récit de Marquis publié dans les Annales de la Bonne Sainte Anne.

Reste la troisième relique, donnée par Marquis à l'église SaintJean-Baptiste. Visiblement gêné, Désilets se met à la recherche d'informations. Il s'adresse alors à la congrégation des Pères du SaintSacrement qui a relayé Tétreau, dès 1902 . A la veille de la publication du Guide, Désilets apprend que l'église possède une relique de sainte Anne, fournie par Marquis, au mois de juillet 1892. Elle provient de Saint-Paul-hors-les-Murs ainsi que l'atteste un certificat d'authenticité signé par Odeschalchi et contresigné par Taschereau ${ }^{35}$. À la lumière de

33 ASN. Succession Marquis. F. Tétreau à Marquis, 9 août 1893.

34 ASN. Succession Marquis. F. Tétreau à Marquis, Arthabaska, 8 avril 1901.

35 Archives de la paroisse de Saint-Célestin. La Tour des Martyrs. Lettre au R. P. P.-H. Hayes, S.S.S., Montréal, sans date. 
ces nouvelles indications, Désilets rédige une note qui confirme la présence, à New York, d'une relique différente de celle de Tétreau et différente également de la troisième dont parle Girard. Il affirme qu'elle y fut apportée par Marquis, à la suite d'une demande empressée des fidèles, fortement émus par les événements miraculeux. La relique qu'il leur remet fut acquise en 1882, elle provient de la chapelle des Odeschalchi et les lettres d'authenticité portent la signature du cardinal Taschereau.

Ainsi qu'on s'en rend compte, il est malaisé d'accompagner Désilets dans la construction de son histoire. L'état de la documentation explique nos difficultés à le suivre, mais en partie seulement. Pour comprendre les raisons qui l'ont amené à rédiger un tel exposé, il faut examiner les objectifs du promoteur. Celui-ci vise moins à trouver la vérité historique qu'à donner crédit et confiance dans les reliques de sainte Anne, conservées à la Tour des Martyrs. Il pense que l'affirmation de leurs origines romaine et aptoise, de même que l'attestation de l'existence de lettres d'authenticité signées par des hommes d'Église aussi prestigieux qu'Odeschalchi et Taschereau concourent à la véracité.

\section{$B$ - L'authenticité des reliques}

L'auteur du Guide convient que le scepticisme peut gagner le visiteur placé devant les 226 reliquaires contenus dans la chapelle. En conséquence, il s'applique à monter un dossier de preuves irréfutables sur l'authenticité des reliques. Ces preuves reposent sur l'exécution d'un travail soigné et précis, toujours en accord avec les règles du droit canonique, effectué sous la surveillance étroite d'un délégué de l'évêque qui, en la matière, détient l'autorité. Ce travail est en effet indispensable, car une relique sans certificat d'authenticité - les «authentiques» - appelé aussi lettres testimoniales, ne peut être exposée à la vénération publique. Dans le cas des reliques nicolétaines, la démonstration s'appuie sur cette stricte observance du droit canonique qui permet l'exposition des reliques lorsque l'ancienneté du culte - la tradition - l'autorise, ou encore lorsqu'il existe déjà des lettres d'authenticité. La preuve en ce qui concerne les reliques du sanctuaire de la Tour des Martyrs paraît reposer sur la possession de lettres testimoniales antérieures.

Dans le diocèse de Nicolet, Calixte Marquis prépare et signe les actes d'authenticité. Il se sert d'un formulaire imprimé sur lequel apparaît son nom ainsi que ses titres: Joseph Calixte Marquis, prêtre, protonotaire apostolique, prélat domestique, chanoine de Lorette, fondateur de la Congrégation des Soeurs de l'Assomption. Le document (voir en annexe) fournit ensuite l'identification de la relique, ici, un cheveu de Marie, mère de Jésus; puis il décrit le reliquaire dans lequel le cheveu 
est renfermé, une boîte (ou coffre) de couleur or et de forme ovale. La lettre testimoniale de telle relique porte la date du 19 avril 1904 et est reconnue et acceptée par François-Xavier Cloutier, évêque de TroisRivières, qui en autorise la vénération dans son diocèse. À chaque relique son authentique, peut-on dire. Mais il semble bien que lorsque les Soeurs Grises arrivent dans la maison de Marquis, à Saint-Célestin, ce n'est pas le cas. Les reliques s'y trouvent partout «dans le salon, dans sa chambre, dans la cuisine au besoin». Selon soeur Saint-Antoine, au mois d'avril 1904, les religieuses font «de minutieuses recherches [...] afin de s'assurer que tout était en bon ordre», car elles craignent que la santé déclinante de Marquis ne les prive «d'explications nécessaires». Celui-ci remplit «une multitude de blancs d'authentiques» contenant «des milliers de noms de saints et de saintes. Aussi un assez grand nombre de reliques de la Passion de Notre-Seigneur».

Les responsables du sanctuaire de la Tour des Martyrs ne lésinent pas pour faire en sorte que chaque relique soit enchassée dans les meilleures conditions et accompagnée du certificat d'authenticité. Afin de démontrer le respect des règles du droit canonique, Le Guide avoue que nombre de reliques ont été inhumées faute d'avoir un caractère d'authenticité affirmé. Après le décès de Marquis, l'évêque Herman Brunault charge F.-A. St-Germain d'apposer les sceaux aux reliquaires. Par la suite, chaque fois qu'un reliquaire exige une réparation, ce qui entraîne le bris du sceau, on fait appel à lui; ainsi, le 6 octobre 1916, le 9 juin 1921 et encore le 19 décembre 1931. Cette année-là, les Soeurs Grises ont réparé plus de trente reliquaires.

Selon Henri Belcourt, curé de la paroisse de Saint-Célestin et responsable du sanctuaire, «il était grand temps d'y voir» ${ }^{36}$. Belcourt se montre particulièrement préoccupé de l'entretien des reliquaires et de leur réfection. Se plaint-on de son attention scrupuleuse, il affirme qu'il n'est pas trop sévère et ne croit pas devoir observer une autre règle de conduite. Dans le but de prévenir la détérioration des reliquaires, Belcourt recommande à l'évêché de les faire visiter une fois l'an et de les réparer au besoin. Les nouvelles procédures vont exiger la présence de l'envoyé épiscopal qui aura «un peu de besogne à faire et [...] probablement plusieurs choses embarrassantes à étudier, en tout cas à [...] soumettre» aux autorités diocésaines.

La réparation des reliquaires se poursuit en 1932: plus de cent reliquaires sont déscellés. Il semble bien que plusieurs ne sont plus convenables, ce qui amène à les changer. Par ailleurs, plusieurs «authentiques» sont corrigées, car les feuillets ont été mal remplis, le vérificateur «s'étant contenté d'inscrire quelques noms seulement pour terminer l'énumération par: etc., etc. ${ }^{37}$ Le délégué de l'évêque exige

\footnotetext{
36 AEN. Cahier de la paroisse de Saint-Célestin, Belcourt à Brunault, 23 et 30 mars 1931 .

37 AEN. Cahier de la paroisse de Saint-Célestin, Belcourt à Brunault, 10 novembre 1932.
} 
alors que tous les noms apparaissent sur le certificat. Les choses se gâtent lorsque F.-A. St-Germain est empêché par la maladie de terminer son travail. Il laisse plusieurs reliquaires sans sceau, alors que des «authentiques» corrigées ne conviennent plus. Pour Belcourt, cet arrêt risque de provoquer une situation fâcheuse. En effet, il croit que les travaux incomplets, ainsi qu'ils apparaissent, peuvent susciter de nombreux doutes sur «la valeur réelle de ce riche trésor». En conclusion des remarques adressées à son supérieur hiérarchique, Belcourt demande la nomination d'un prêtre autorisé à sceller les reliquaires et «à approuver ou désapprouver le travail qui restera à faire». Le 26 novembre 1932, l'évêque de Nicolet remplace St-Germain par le chanoine Joseph Bourgeois, professeur de droit canonique au séminaire. Il croit que rien ne doit être négligé, car, écrit-il, «il s'agit de fortifier la croyance de notre peuple à l'authenticité de nos saintes reliques et d'attirer davantage les foules à notre pieux sanctuaire de Saint-Célestin» ${ }^{38}$.

Grâce à ce travail, entrepris du vivant même de Marquis, le fouillis constaté par les Soeurs Grises, à leur arrivée, fait place vers 1930, à un corpus d'environ 6200 reliques dûment «authentiquées», impeccablement rassemblées dans 226 reliquaires marqués d'un sceau et disposés dans la nouvelle chapelle de la Tour des Martyrs. Devant ces murs littéralement «couverts d'ossements», selon les termes utilisés dans Le Guide, le pèlerin est tour à tour étonné, recueilli et dévot à l'endroit des intercesseurs de qui il attend des merveilles. De l'aveu de l'évêque Brunault qui, en 1930, bénit la nouvelle chapelle, il s'y passe «quelque chose d'extraordinaire» ${ }^{39}$.

\section{3 - LE MERVEILLEUX}

Pas de pèlerinage sans reliques, pas de pèlerinage non plus sans eau: fontaine et source qui fournissent l'eau de la régénération et de la guérison. Le voisinage des reliques et du puits procure à l'eau sa vertu guérisseuse $^{40}$, et provoque ainsi le merveilleux, le miraculeux, les faits exceptionnels. Ceux-ci comptent assurément parmi les raisons qui expliquent la naissance et la survie d'un pèlerinage ${ }^{41}$. Les Rédemptoristes le savent assez pour éditer, en 1907, un «recueil de guérisons miraculeuses opérées par l'intercession de la Bonne Sainte Anne de Beaupré» ${ }^{42}$. Les récits des miracles relatés dans les annales des pèlerinages canadiens et la rubrique des «faveurs obtenues» qui s'y trouvent visent aussi à démontrer l'efficacité du pèlerinage.

\footnotetext{
38 AEN. Cahier de la paroisse de Saint-Célestin, Brunault à Belcourt, 26 novembre 1932.

39 Arthur Girard, La Tour des Martyrs de Saint-Célestin..., 92.

40 Pierre-André Sigal, L'homme et le miracle dans la France médiévale, XIe-XIIe siècles (Paris, Cerf, 1985), 54-55.

41 Benoît Lacroix et Jean Simard, dir., Les pèlerinages au Québec, 1.

42 Les miraculés de la Bonne Sainte Anne (Québec, P. Larose, imprimeur, 1907), 277 p.
} 


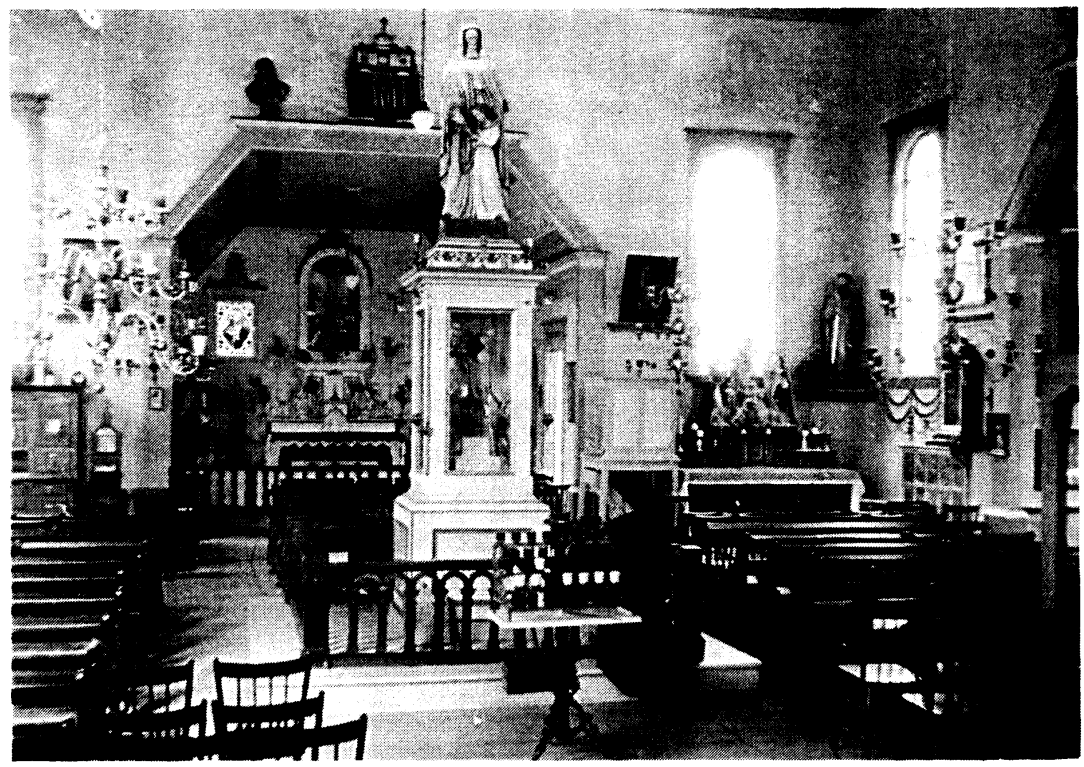

Intérieur de la Vieille Tour ou chapelle Sainte-Anne. La relique du bras de sainte Anne y était exposée.

Archives des Soeurs Grises de Nicolet.

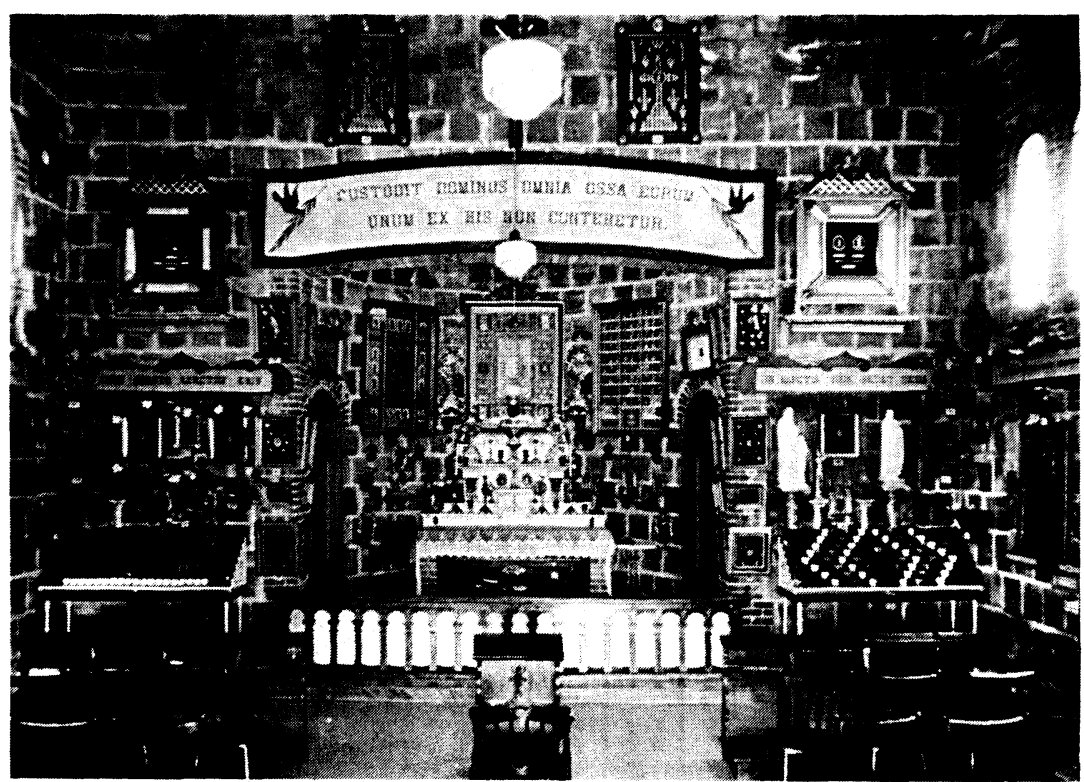

Intérieur de la Tour des Martyrs; la translation des reliques de la vieille à la nouvelle Tour fut faite en 1930.

Archives des Soeurs Grises de Nicolet. 
Le clergé nicolétain ne pense pas autrement. Aussi, la question de l'intercession des saints de la Tour des Martyrs est-elle cruciale et doitelle être soulevée, ce que fait Le Guide. "S'opère-t-il des miracles à la Tour des Martyrs?» Réponse: «Il ne s'en est encore constaté aucun au sens théologique et canonique du mot.» Mais le rédacteur dénonce l'incrédulité, le manque de confiance, voire l'outrance de quiconque exige des prodiges. Il est cependant impossible de rester sur ce constat peu encourageant. Demander la patience aux «braves populations si riches de foi ferme et éclairée envers les saints intercesseurs» n'a pas non plus une grande valeur de conviction. Pourtant, la réponse, en apparence tranchée, ne rend pas un verdict sans appel. N'y a-t-il pas des témoignages de plus en plus nombreux de la part de gens ayant obtenu qui, la guérison, qui, le soulagement de la maladie, qui, une consolation ${ }^{43}$ ? Et pourquoi ne pas faire confiance à sainte Anne qui a «gâté la bonne population canadienne en faisant éclater [...] des prodiges de puissance et de bonté?» Sainte Anne dont la Tour des Martyrs conserve plusieurs reliques; sainte Anne dont le nom est donné à un puits légendaire.

Un prodige survenu lors de la construction de la chapelle, à proximité de laquelle se trouve un puits désaffecté, est à l'origine de cette légende, racontée par Désilets. Accompagnés de soeur Saint-Antoine, les ouvriers s'approchent du puits et constatent la présence d'une eau boueuse, impropre à la construction. La religieuse revient interroger Calixte Marquis qui les retourne au puits. «Une chaudière est descendue dans le puits, qui en remonte avec une eau claire comme si on l'avait puisée entre des rochers.» Depuis ce moment, sur la décision de Marquis, l'eau du puits, désormais nommé puits de sainte Anne, ne doit plus servir qu'à boire ou laver les malades et les infirmes. Marquis sait bien que l'eau est un ingrédient précieux pour le succès d'un pèlerinage ${ }^{44}$. Désilets, promoteur du pèlerinage de la Tour des Martyrs, le sait également, d'où l'invention de la légende qui repose sur le récit de soeur Saint-Antoine $e^{45}$.

Les religues s'avèrent un second ingrédient pour l'invention d'un pèlerinage. A Saint-Célestin, un os de l'avant-bras de sainte Anne, renfermé dans un reliquaire brachial est la plus prestigieuse de toutes. Prestige qu'elle tient de son association avec les autres ossements acquis

43 ASGN. Guérisons. Une liste dressée à partir de la chronique de l'hospice Sainte-Anne de Saint-Célestin donne quelques informations sur dix-huit faits «merveilleux» entre le 13 juin 1898 et le 9 octobre 1933 .

44 Robert Pannet, Marie au buisson ardent (Paris, SOS, 1982), 81; Brigitte Caulier, «Espace sacré et cultes thérapeutiques autour des fontaines en France», F. Lebrun et N. Séguin, Sociétés villageoises et rapports villes-campagnes dans la France de l'Ouest, XVIIe-XXe siècles (TroisRivières, Centre d'études québécoises, 1987), 285-295.

45 La religieuse n'arrive à Saint-Célestin qu'en 1900, soit deux ans après la construction de la chapelle. Elle-même spécifie bien qu'elle tient ces faits de la bouche de l'ouvrier en charge des travaux, se limitant à le citer fidèlement. 


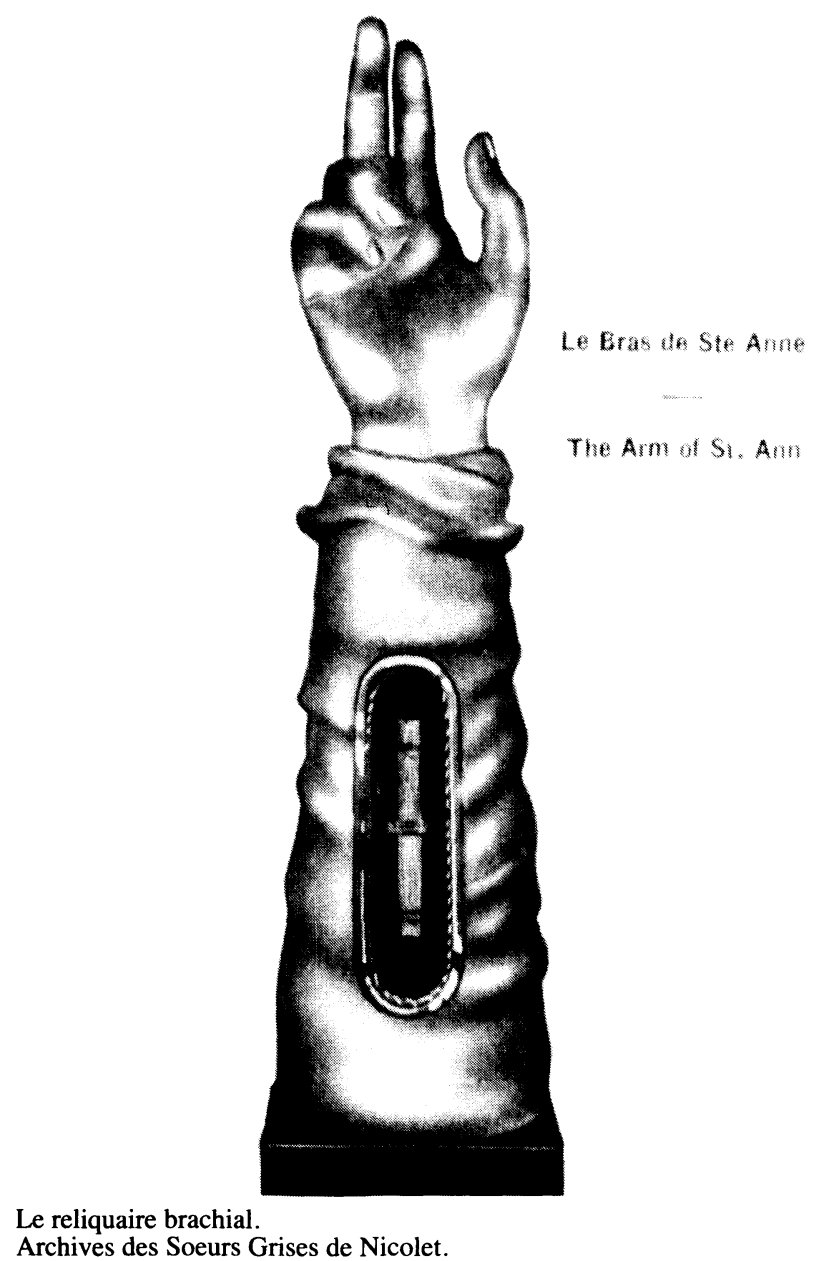

par Marquis, et plus particulièrement avec la relique qu'il obtient en faveur du pèlerinage de Sainte-Anne-de-Beaupré. Le Guide raconte que, le 1er mai 1892, de retour d'Europe, Calixte Marquis débarque à New York et se rend chez Frédéric Tétreau, curé de l'église Saint-Jean-Baptiste. À la demande du prêtre, la relique de sainte Anne est exposée pendant trois semaines. «Les foules accourent, vénèrent la sainte Relique et l'acclament. Deux miracles éclatent.»

La connaissance des faits miraculeux survenus à New York est due à la chronique de Bernard $\mathrm{O}$ 'Reilly publiée d'abord dans le journal L'Univers et reprise dans Les Annales de la Bonne Sainte-Anne-deBeaupré. Avant cela, Le Courrier du Canada qui, lui, puise son information dans The New York Herald, note le mouvement de dévotion 
déclenché par l'exposition de la relique ${ }^{46}$. Grâce à ces annales et au clergé, on peut penser que la nouvelle est amplement diffusée. À titre d'exemple, voici ce que raconte Charles Bellemare, curé de Saint-Boniface-de-Shawinigan, à un homologue français, en 1893.

En passant à New York, il (Marquis) a parlé à M. Tétreau de ce précieux dépôt qu'il apporta au Canada; le curé demande comme faveur spéciale d'en faire profiter ses paroissiens et, sans avis préalable, il s'en va avec Mgr Marquis à son église, le dimanche à l'office des vêpres, annonce que l'on va vénérer une relique de sainte Anne. Un épileptique se présente avec les autres et avant d'arriver à la Sainte Table, il tombe dans des convulsions épouvantables. M. Tétreau traverse la foule, lui applique la relique; immédiatement, cet homme se calme, il est guéri. La nouvelle s'en répand comme l'éclair dans tout New York; Mgr Marquis doit rester 2 ou 3 semaines pour satisfaire la piété des chrétiens de cette ville qui remercient sainte Anne des faveurs obtenues par des dons à l'Église des Canadiens. Ces dons, dans ce court espace de temps, donnèrent une somme fabuleuse d'une vingtaine de mille dollars. Un autel fut élevé à sainte Anne et encore maintenant, les pèlerins arrivent en foule demander à sainte Anne protection et guérison. Pendant que j'étais là, j'eus la faveur de vénérer et de faire vénérer la relique que $\mathbf{M}$. Tétreau a obtenue et qui est continuellement exposée $[\ldots . . .]^{47}$

L'émerveillement de Charles Bellemare est corroboré par les récits des guérisons que l'on peut lire dans sa correspondance et dans «le recueil de guérisons miraculeuses [...] ${ }^{48}$ dans lequel il signe d'ailleurs la relation d'un miracle. Cette croyance au miracle n'appartient pas qu'au «brave» curé de campagne, comme le prouvent les Mémoires de Lionel Groulx ${ }^{49}$. Ses pèlerinages à Lourdes et à Lisieux le montrent en attente du fait merveilleux. Il compte parmi ses amis l'abbé Antonio Hébert, un miraculé de Sainte-Anne-de-Beaupré, «guéri vers les vingt ans d'une ostéite au genou qui menaçait de lui fermer l'accès au sacerdoce $[\ldots]\rangle^{50}$.

Ces relations de Charles Bellemare et le fait exceptionnel dont parle le chanoine Groulx sont à rapprocher des événements prodigieux

46 Bernard O'Reilly, «Les miracles de la Bonne Sainte Anne à New York», Annales de la Bonne Sainte Anne (janvier 1893): 185-189. Prêtre séculier, puis jésuite, O'Reilly est un ami de longue date de Calixte Marquis avec qui il a fait ses études.

47 N.-J. Chaline, R. Hardy et J. Roy, La Normandie et le Québec vus du presbytère, documents annotés et présentés (Montréal et Rouen, Boréal et Presses de l'Université de Rouen), 171-172. D'après le récit de soeur Saint-Antoine, Marquis et Tétreau se sont entendus pour partager «à l'amiable, au profit de nos oeuvres religieuses, les aumônes et les dons pieusement offerts à la bonne sainte Anne par ses heureux protégés».

48 Les miraculés de la Bonne Sainte Anne (Québec, P. Larose, imprimeur, 1907), 127-128.

49 Benoît Lacroix, La religion de mon père (Montréal, Bellarmin, 1986), 306 p. Voir «Lionel Groulx et ses croyances», 199-218.

so Lionel Groulx, Mes mémoires (Montréal, Fides, 19??), tome 1: 1878-1920: 175-286. Sur la croyance des Québécois au miracle à la fin du XIXe siècle, voir Serge Gagnon, Le Québec et ses historiens (Québec, Presses de l'Université Laval, 1978), 43ss. 
rapportés dans des lettres adressées soit à la supérieure des Soeurs Grises, soit au curé de Saint-Célestin. Dans les deux exemples qui suivent, notons que sainte Anne occupe la place centrale du récit et que la guérison est parfois obtenue à la suite de l'attouchement de la relique, parfois après que le malade se soit lavé avec l'eau miraculeuse.

Le 20 mai 1925, A. P. porte son enfant âgé de deux ans et demi à la Tour des Martyrs. Incapable de marcher à la suite d'une chute, l'enfant est, selon deux médecins, virtuellement condamné à une infirmité définitive. Le malheureux père fait part de son infortune au curé qui s'adresse à sainte Anne. Il pose ensuite la relique de la grande thaumaturge sur la partie malade. Confiant, le père retourne chez lui et revient, comme promis, neuf dimanches consécutifs. Puis, un jour, après un nouvel attouchement de la relique, le père a l'idée d'appuyer l'enfant sur la balustrade qui entoure la statue et le précieux reliquaire. Voilà que tout à coup l'enfant, "tout en boitant un peu, fait le tour de la balustrade» et se rend jusqu'à la porte extérieure. Dans une autre lettre, au mois de septembre 1930, Belcourt, curé de la paroisse de Saint-Célestin, reçoit le récit d'un pèlerin de la paroisse de SainteEulalie. Celui-ci raconte qu'il souffre, depuis plus d'un mois, d'une plaie à une jambe ce qui, bien sûr, l'empêche de travailler. S'étant lavé avec «l'eau de sainte Anne», il est guéri.

Les autorités ecclésiastiques ne reproduisent pas ces cas. Dans $L e$ Guide, Désilets affirme qu'il ne s'agit pas d'événements confrontés à l'expertise médico-théologique. En 1932, lors de sa publication, aucune preuve ne permet de les présenter comme des faits miraculeux. Mais, en revanche, on reçoit plusieurs témoignages sur l'utilité de l'intercession des saints. La rubrique des faveurs obtenues, publiée dans Les Annales de la Tour des Martyrs de Saint-Célestin ${ }^{51}$ a pour fonction de propager ces nouvelles afin de stimuler la dévotion. Reste que «l'extraordinaire» dont parle l'évêque Brunault est à venir.

\section{CONCLUSION}

Le pèlerinage de la Tour des Martyrs de Saint-Célestin est une invention de prêtres qui désirent développer la piété populaire. On utilise à cette fin un lieu déjà consacré par la présence de nombreuses reliques, la chapelle, jadis construite par Calixte Marquis pour les offrir à la vénération publique. Dans ce cas-ci, la relique a donc créé le pèlerinage. Ailleurs, au Québec, c'est une statue: celle de Notre-Dame à Lac Bouchette (1912), celles du Sacré-Coeur (1916) et de Notre-Dame (1930) à Beauvoir, près de Sherbrooke, pour ne citer que ces exemples.

51 Jean Roy, «Un intermédiaire culturel: Les Annales de la Tour des Martyrs de SaintCélestin, au Québec (1931-1952)», Annales de Bretagne et des pays de l'Ouest, 95,4 (1988): 499510. 
Dans chaque lieu, un objet physique concret polarise la piété: le tombeau de Kateri Tekakwita, la relique de sainte Anne à Saint-Anne-deBeaupré et à Saint-Célestin, la statue de Notre-Dame-de-Liesse, rapportée de France, intronisée au Gésu en 1878. La piété ultramontaine, comme ces exemples l'illustrent, est éminemment sensorielle.

Or, la relique paraît être un objet symbolique peu efficace pour lancer un pèlerinage ${ }^{52}$. Moins, en tout cas, que l'histoire qui a pour rôle de lui accorder une légitimité, moins aussi que le récit merveilleux à l'origine de sa crédibilité.

Sur Calixte Marquis, il y a peu à ajouter. Ses rapports avec les évêques québécois fluctuent; un temps proche d'Elzéar Taschereau, à Québec, on finit par prendre des distances avec lui; écouté d'abord par son évêque, Marquis s'estime isolé par Elphège Gravel à qui d'ailleurs il ne rend pas la vie facile. Quant à ses relations avec Laflèche, les deux se détestent cordialement. Mais nul n'est plus connu que lui dans Nicolet, et rien ne lui est étranger tant il est mêlé à tout: colonisation, politique, éducation et, bien sûr, religion. Il connaît le succès et il a des amis. Tous reconnaissent sa piété et sa dévotion aux saintes reliques. Oubliant le côté acrimonieux de son caractère, les promoteurs du sanctuaire s'efforcent de faire ressortir sa dévotion à l'endroit des saints et son attachement aux reliques qu'il a acquises. Grâce à lui, celles-ci sont offertes au culte, non seulement à Saint-Célestin mais partout au Québec, car Marquis en donne un grand nombre, faisant de Saint-Célestin la plaque tournante de leur diffusion, celles de sainte Anne en particulier, dont l'efficacité paraît facile à démontrer. Nul doute, selon lui, que le prodige de l'eau miraculeuse du puits est dû à la mère de Marie. L'anecdote religieuse est ici bien utilisée pour fonder le mérite du pèlerinage. Elle augmente aussi le crédit de Marquis. La relation des faits miraculeux survenus dans l'église Saint-Jean-Baptiste de New York sert à renforcer la croyance et accrédite, par assimilation, la principale relique de sainte Anne conservée à Saint-Célestin.

La promotion réussit. De modeste lieu de pèlerinage paroissial, la Tour des Martyrs jouit en 1933 d'une belle renommée et est reconnue «Sanctuaire national de la dévotion aux Reliques de Saints». Moins de trente ans plus tard, le pèlerinage est abandonné, comme plusieurs autres, il est vrai, qui sont nés à la même période ${ }^{53}$. Il est également vrai qu'il n'offre pas tous les attraits d'un grand pèlerinage comme celui de SainteAnne-de-Beaupré, qu'il ne s'y produit aucun miracle attesté par l'Église, que le clergé montre finalement une certaine tiédeur, à moins que ce ne

\footnotetext{
52 Robert Pannet, op. cit., 69.

53 Jean Roy, «Mutation et déclin de la solidarité religieuse: la fréquentation de la Tour des Martyrs de Saint-Célestin», Roger Levasseur, dir., De la Sociabilité, spécificité et mutations (Montréal, Boréal, 1990), 133-135.
} 
soit une «certaine peur du ridicule» ${ }^{54}$, avant que les fidèles ne manifestent une désaffection qu'il faut maintenant tenter d'expliquer.

L'oeuvre de la Tour des Martyrs est une riposte du clergé à la montée «de l'effrayante marée matérialiste» contre laquelle il faut défendre les fidèles. C'est ainsi qu'Arthur Girard introduit la Tour des Martyrs de Saint-Célestin, symbole des forces spirituelles dans le monde. À sa façon, la création du pèlerinage peut servir à illustrer la lutte de l'Église québécoise contre la modernité, contre le changement que vit la société québécoise au cours de ces années de crise économique qui, à leur manière, marquent la modernité 55 .

La «marée matérialiste» n'épargne pas les campagnes qui, elles aussi, bougent. L'intérêt manifesté dans les paroisses nicolétaines à l'endroit des divers moyens de l'enseignement agricole en témoigne largement. Le clergé nicolétain, issu du monde rural, proche de ses ouailles, les stimule et les aide à sortir l'agriculture de ses difficultés, les amenant de la sorte à exercer une meilleure maitrise de la nature ${ }^{56}$. La présence des spécialistes nouveaux ou plus nombreux comme le médecin, le vétérinaire et l'agronome, finit d'arracher au clergé son monopole du savoir, contribuant à affaiblir, comme on l'a observé en Bretagne, la capacité de la religion à guider l'individu, le groupe et la société ${ }^{57}$.

Suivant cette interprétation, le clergé crée le pèlerinage de la Tour des Martyrs pour la dévotion aux saintes reliques, alors que, dès le début du XXe siècle, des voix autorisées s'élèvent, non contre le culte des reliques, mais contre les reliques douteuses ou superstitieuses. Certes, l'épiscopat nicolétain voit à l'application rigoureuse du code de droit canonique de 1917 qui accorde une grande importance à la question de l'authenticité des reliques ${ }^{58}$. En conséquence, seules celles dont l'authenticité s'appuie sur une vénération ancienne, prouvée par les lettres testimoniales, sont offertes à la dévotion.

En dépit des tendances canoniques au sujet des reliques, le clergé nicolétain invente le pèlerinage de la Tour des Martyrs. De plus, il le fait dans une conjoncture de crise qui, redisons-le, paraît favorable à l'épiscopat nicolétain. Mais, c'est aussi un temps de rupture, avant le changement. Le désintéressement des fidèles finit bientôt par l'emporter sur l'obstination d'une partie du clergé qui, faut-il le souligner, ne ménage pas la propagande.

\footnotetext{
54 Nicole Hermann-Mascard, op. cit., préface de Jean Imbert, 7.

55 Arnaldo Nesti, «Églises et modernité», Social Compass, 34,2-3 (1987): 135-136.

56 Jean Roy, «L'enseignement agricole au Québec, 1926-1964». Communication présentée au colloque franco-québécois Reproduction sociale en milieu rural en contexte d'urbanisation. À paraître.

Yves Lambert, Dieu change en Bretagne (Paris, Cerf, 1985), 451 p. Étude de la religion à Lémerzel de 1900 à nos jours. Voir aussi André Burguière, Bretons de Plozevet (Paris, Flammarion, 1977), $395 \mathrm{p}$.

58 Nicole Hermann-Mascard, op. cit., 8.
} 